\title{
Monopoles and Modifications of Bundles over Elliptic Curves ${ }^{\star}$
}

\author{
Andrey M. LEVIN ${ }^{\dagger \ddagger}$, Mikhail A. OLSHANETSKY ${ }^{\dagger \S}$ and Andrei V. ZOTOV ${ }^{\dagger \S}$ \\ $\dagger$ Max Planck Institute of Mathematics, Bonn, Germany \\ $\ddagger$ Institute of Oceanology, Moscow, Russia \\ E-mail: alevin@wave.sio.rssi.ru \\ $\S$ Institute of Theoretical and Experimental Physics, Moscow, Russia \\ E-mail: olshanet@itep.ru,zotov@itep.ru
}

Received November 20, 2008, in final form June 10, 2009; Published online June 25, 2009

doi:10.3842/SIGMA.2009.065

\begin{abstract}
Modifications of bundles over complex curves is an operation that allows one to construct a new bundle from a given one. Modifications can change a topological type of bundle. We describe the topological type in terms of the characteristic classes of the bundle. Being applied to the Higgs bundles modifications establish an equivalence between different classical integrable systems. Following Kapustin and Witten we define the modifications in terms of monopole solutions of the Bogomolny equation. We find the Dirac monopole solution in the case $R \times$ (elliptic curve). This solution is a three-dimensional generalization of the Kronecker series. We give two representations for this solution and derive a functional equation for it generalizing the Kronecker results. We use it to define Abelian modifications for bundles of arbitrary rank. We also describe non-Abelian modifications in terms of thetafunctions with characteristic.
\end{abstract}

Key words: integrable systems; field theory; characteristic classes

2000 Mathematics Subject Classification: 14H70; 14F05; 33E05; 37K20; 81R12

\section{Introduction}

The modifications (or the Hecke transformation) of bundles over complex curves is a correspondence between two bundles $E$ and $\tilde{E}$. It is isomorphism in a complement of some divisor. A modification can change the topological type of the original bundle. From the field-theoretical point of view the modification is provided by a gauge transformation of sections, which is singular at the divisor. In [1] we apply this procedure to the Higgs bundles. The Higgs bundles are the phase spaces of the Hitchin integrable systems [2]. Modifications acts on the phase space as a symplectic transformation. In this special case we call the modification the Symplectic Hecke Correspondence. For the Higgs bundles over elliptic curves with marked points Symplectic Hecke Correspondence leads to a symplectomorphism between different classical integrable systems such as

- Elliptic Calogero-Moser system $\Leftrightarrow$ Elliptic GL $(N, \mathbb{C})$ Top, [1];

- Calogero-Moser field theory $\Leftrightarrow$ Landau-Lifshitz equation, [1, 4];

- Painlevé VI $\Leftrightarrow$ non-autonomous Zhukovsky-Volterra gyrostat, [3].

* This paper is a contribution to the Proceedings of the Workshop "Elliptic Integrable Systems, Isomonodromy Problems, and Hypergeometric Functions" (July 21-25, 2008, MPIM, Bonn, Germany). The full collection is available at http://www.emis.de/journals/SIGMA/Elliptic-Integrable-Systems.html 
In these examples modifications increase the degree of the underlying bundles on one. In general, modifications act as the Bäcklund transformations of integrable systems. If degree of the bundles (modula rank) is not changed then modifications produce what is called the autoBäcklund transformations. It turned out that the modification in the first example is equivalent to the twist of $R$-matrices $[5,6]$ that transforms the dynamical $R$-matrices of the IRF models of the GL $(N)$ type [7] to the vertex $R$-matrices [8] corresponding to the $\mathrm{GL}(N)$ generalization of the XYZ models.

The modifications are parameterized by vectors $\vec{m}$ of the weight lattices $P$ of $\operatorname{SL}(N, \mathbb{C})$. If $\vec{m}$ belongs to the root sublattice $Q \subset P$, then the modified bundle $\tilde{E}$ has the same degree as $E$. Otherwise, the degree of bundle is changed. The modifications can be described by changing another topological invariant. It is a characteristic class of a bundle. Let the base of $E$ be a Riemann surface $\Sigma_{g}$ of genus $g$. Then the characteristic class of $E$ is an element of $H^{2}\left(\Sigma_{g}, \mathbb{Z}_{N}\right) \sim \mathbb{Z}_{N}$, where $\mathbb{Z}_{N} \sim P / Q$ is a center of $\operatorname{SL}(N, \mathbb{C})$. Another example of the characteristic classes, is the characteristic class of spin-bundles, that will not considered here, is the Stiefel-Whitney class $H^{2}\left(\Sigma_{g}, Z_{2}\right)$.

Here we discuss a field-theoretical interpretation of modifications. It was established in [9] that the modifications are related to the Dirac monopole configurations in a topological version of the $\mathcal{N}=4$ four-dimensional super-symmetric Yang-Mills theory. If "the space-time" of the topological theory has the form $\mathbb{R}^{2} \times \Sigma_{g}$, then the modifications of $E$ over $\Sigma_{g}$ are parameterized by the monopoles charges.

To describe the modification it is sufficient to neglect the "time" dependence and consider $\mathbb{R} \times \Sigma_{g}$. The condition for fields to preserve the supersymmetry amounts to the Bogomolny equation.

The aims of this paper are

- To define modifications and describe their interrelations with the Bogomolny equation following [9]. We consider a special configuration of the space-time $\mathbb{R}^{2} \times \Sigma_{\tau}$, where $\Sigma_{\tau}$ is an elliptic curve with the modular parameter $\tau$.

- To find solutions of the Bogomolny equation in the case of line bundles over $\Sigma_{\tau}$. They are generalizations of the Kronecker series [17]. We give two representations of the solution and prove their equivalence by means of the functional equation generalizing the Kronecker functional equation.

- To describe non-Abelian modifications that are not related directly to solutions of the Bogomolny equation and follows from our previous results.

\section{Characteristic classes of holomorphic bundles over complex curves}

We describe holomorphic bundles over complex curves $\Sigma_{g}$ of genus $g$ and define their characteristic classes.

\subsection{Global description}

Let $\pi_{1}\left(\Sigma_{g}\right)$ be a fundamental group of $\Sigma_{g}$. It has $2 g$ generators $\left\{a_{\alpha}, b_{\alpha}\right\}$, corresponding to the fundamental cycles of $\Sigma_{g}$ with the relation

$$
\prod_{\alpha=1}^{g}\left[a_{\alpha}, b_{\alpha}\right]=1,
$$

where $\left[a_{\alpha}, b_{\alpha}\right]=a_{\alpha} b_{\alpha} a_{\alpha}^{-1} b_{\alpha}^{-1}$ is the group commutator. 
Let $\rho$ be a representation of $\pi_{1}$ in $\mathbb{C}^{N}$. Consider a holomorphic adjoint $\operatorname{GL}(N, \mathbb{C})$ bundle $E$ over $\Sigma_{g}$. In fact, $E$ is a $\operatorname{PGL}(N, \mathbb{C}) \sim \operatorname{PSL}(N, \mathbb{C})$ bundle, because the center of $\operatorname{GL}(N, \mathbb{C})$ does not act in the adjoint representation. The bundle $E$ can be defined by holomorphic transition matrices of its sections $s \in \Gamma(E)$ around the fundamental cycles. Let $z \in \Sigma_{g}$ be a fixed point. Then

$$
s\left(a_{\alpha} z\right)=\rho\left(a_{\alpha}\right) s(z), \quad s\left(b_{\beta} z\right)=\rho\left(b_{\beta}\right) s(z) .
$$

Due to $(2.1)$ we have

$$
\prod_{\alpha=1}^{g}\left[\rho\left(a_{\alpha}\right), \rho\left(b_{\alpha}\right)\right]=\mathrm{Id} .
$$

Let $\mathcal{K}$ be an extension of $\pi_{1}$ by the cyclic group $\mathbb{Z}_{N} \sim \mathbb{Z} / N \mathbb{Z}$

$$
1 \rightarrow \mathbb{Z}_{N} \rightarrow \mathcal{K} \rightarrow \pi_{1}\left(\Sigma_{g}\right) \rightarrow 1
$$

The group $\mathcal{K}$ is defined by the relation

$$
\prod_{\alpha=1}^{g}\left[a_{\alpha}, b_{\alpha}\right]=\omega, \quad \omega^{N}=1 .
$$

Let $\hat{\rho}$ be a representation of $\mathcal{K}$ in $\operatorname{GL}(N, \mathbb{C})$. Then using $\hat{\rho}$ as transition matrices we define a bundle over $\Sigma_{g}$. But now instead of $(2.2)$ we have

$$
\prod_{\alpha=1}^{g}\left[\hat{\rho}\left(a_{\alpha}\right), \hat{\rho}\left(b_{\alpha}\right)\right]=\omega \mathrm{Id} .
$$

Here $\omega$ Id is the generator of the center $\mathcal{Z}(\operatorname{SL}(N, \mathbb{C})) \sim \mathbb{Z}_{N}$ of $\operatorname{SL}(N, \mathbb{C})$. It means that $\hat{\rho}$ can serve as transition matrices only for $\operatorname{PSL}(N, \mathbb{C})$ bundles, but not for $\operatorname{SL}(N, \mathbb{C})$ or $\operatorname{GL}(N, \mathbb{C})$ bundles. Note, that the fibers of the $\operatorname{PSL}(N, \mathbb{C})$-bundles are spaces of representations with highest weights from the root lattice $Q$ (A.2) including the adjoint representation with the highest weight $\varpi_{1}+\varpi_{N-1}$ (A.3). For the $\operatorname{SL}(N, \mathbb{C})$ representations the highest weights belong to the weight lattice $P(\mathrm{~A} .4)$. In this way elements from the factor group $P / Q \sim \mathcal{Z}(\mathrm{SL}(N, \mathbb{C}))$ (A.6) define an obstruction to lift $\operatorname{PSL}(N, \mathbb{C})$ bundles to $\operatorname{SL}(N, \mathbb{C})$ bundles.

The obstruction has a cohomological interpretation. Consider the exact sequence following from (A.1)

$$
\rightarrow H^{1}\left(\Sigma_{g}, \operatorname{SL}(N, \mathbb{C})\right) \rightarrow H^{1}\left(\Sigma_{g}, \operatorname{PSL}(N, \mathbb{C})\right) \rightarrow H^{2}\left(\Sigma_{g}, \mathcal{Z}(\operatorname{SL}(N, \mathbb{C}))\right) \rightarrow \cdots .
$$

The groups $H^{1}\left(\Sigma_{g}, \operatorname{SL}(N, \mathbb{C})\right), H^{1}\left(\Sigma_{g}, \operatorname{PSL}(N, \mathbb{C})\right)$ are the moduli space of $\operatorname{SL}(N, \mathbb{C})$ and $\operatorname{PSL}(N, \mathbb{C})$ bundles. Then $H^{2}\left(\Sigma_{g}, \mathcal{Z}(\operatorname{SL}(N, \mathbb{C}))\right)$ defines an obstruction to lift $\operatorname{PSL}(N, \mathbb{C})$ bundles to $\operatorname{SL}(N, \mathbb{C})$ bundles. We call $\xi \in H^{2}\left(\Sigma_{g}, \mathbb{Z}_{N}\right)$ the characteristic class of a $\operatorname{PSL}(N, \mathbb{C})$ bundle. In fact, $H^{2}\left(\Sigma_{g}, \mathbb{Z}_{N}\right) \sim \mathbb{Z}_{N}$ and $\omega$ in $(2.4)$ represents $\xi \in H^{2}\left(\Sigma_{g}, \mathbb{Z}_{N}\right)$.

This construction can be generalized to any factor-group $G_{l}=\operatorname{SL}(N, \mathbb{C}) / \mathbb{Z}_{l}$, where $l$ is a nontrivial divisor of $N,(N=p l, l \neq 1, N)$. Consider an extension $\mathcal{K}_{l}$ of $\pi_{1}\left(\Sigma_{g}\right)$ by $\mathbb{Z}_{l}$ (compare with (2.3))

$$
1 \rightarrow \mathbb{Z}_{l} \rightarrow \mathcal{K}_{l} \rightarrow \pi_{1}\left(\Sigma_{g}\right) \rightarrow 1
$$

Let $E_{l}$ be a holomorphic $G_{l}$-bundle. The fibers of $E_{l}$ belong to a irreducible representation of $G_{l}$ with a highest weight $\nu \in \Gamma\left(G_{l}\right)$ (A.7). Then the transition matrices representing $\mathcal{K}_{l}$ satisfy the relation

$$
\prod_{\alpha=1}^{g}\left[\hat{\rho}\left(a_{\alpha}\right), \hat{\rho}\left(b_{\alpha}\right)\right]=\omega^{p} \operatorname{Id}, \quad\left(\omega^{p}\right)^{l}=1 .
$$


It follows from the exact sequence

$$
1 \rightarrow \mathbb{Z}_{l} \rightarrow \mathrm{SL}(N, \mathbb{C}) \rightarrow G_{l} \rightarrow 1,
$$

that elements from $H^{2}\left(\Sigma_{g}, \mathbb{Z}_{l}\right) \sim \mathbb{Z}_{l}$ are obstructions to lift $G_{l}$ bundle $E_{l}$ to a $\operatorname{SL}(N, \mathbb{C})$-bundle. The group $\mathbb{Z}_{l}$ can be identified with the center of the dual group ${ }^{L} G_{l} \sim G_{p}=\operatorname{SL}(N, \mathbb{C}) / \mathbb{Z}_{p}$ (see (A.11) and (A.10)). Thus, the obstructions to lift $G_{l}$ bundles $E_{l}$ to a $\operatorname{SL}(N, \mathbb{C})$ bundles are defined by $H^{2}\left(\Sigma_{g}, \mathcal{Z}\left({ }^{L} G_{l}\right)\right)$.

On the other hand, since $\mathbb{Z}_{p}$ is a center of $G_{l}$ we have the sequence

$$
1 \rightarrow \mathbb{Z}_{p} \rightarrow G_{l} \rightarrow \operatorname{PSL}(N, \mathbb{C}) \rightarrow 1,
$$

where $\mathbb{Z}_{p}$ is a center of $G_{l}$. Then elements from $H^{2}\left(\Sigma_{g}, \mathcal{Z}\left(G_{l}\right)\right)$ are obstructions to lift a $\operatorname{PSL}(N, \mathbb{C})$-bundle to a $G_{l}$-bundle. Summarizing we have defined two types of the characteristic classes

$$
\begin{aligned}
& H^{2}\left(\Sigma_{g}, \mathcal{Z}\left(G_{l}\right)\right) \text { - obstructions to lift a } \operatorname{PSL}(N, \mathbb{C}) \text { bundle to a } G_{l} \text { bundle, } \\
& H^{2}\left(\Sigma_{g}, \mathcal{Z}\left({ }^{L} G_{l}\right)\right) \text { - obstructions to lift a } G_{l} \text { bundle to a } \operatorname{SL}(N, \mathbb{C}) \text { bundle. }
\end{aligned}
$$

Though for $\omega \neq 1 \operatorname{PSL}(N, \mathbb{C})$ bundles cannot be lifted to $\operatorname{SL}(N, \mathbb{C})$ bundles, they can be lifted to $\operatorname{GL}(N, \mathbb{C})$ bundles. From the exact sequence

$$
1 \rightarrow \mathcal{O}^{*} \stackrel{\operatorname{det}}{\rightarrow} \mathrm{GL}(N, \mathbb{C}) \rightarrow \operatorname{PGL}(N, \mathbb{C}) \rightarrow 1
$$

we have

$$
H^{1}\left(\Sigma_{g}, \operatorname{GL}(N, \mathbb{C})\right) \rightarrow H^{1}\left(\Sigma_{g}, \operatorname{PGL}(N, \mathbb{C})\right) \rightarrow H^{2}\left(\Sigma_{g}, \mathcal{O}^{*}\right)
$$

The Brauer group $H^{2}\left(\Sigma_{g}, \mathcal{O}^{*}\right)$ vanishes and therefore, there is no obstruction to lift $\operatorname{PGL}(N, \mathbb{C}) \sim$ $\operatorname{PSL}(N, \mathbb{C})$ bundles to $\mathrm{GL}(N, \mathbb{C})$ bundles. We will demonstrate it below.

\subsection{Holomorphic bundles over elliptic curves}

We define an elliptic curve $(g=1)$ as the quotient $\Sigma_{\tau}=\mathbb{C} /(\mathbb{Z}+\tau \mathbb{Z})$. In this case we can construct explicitly the generic transition matrices for $G_{l}$-bundles.

The curve has two fundamental cycles $a:(z \rightarrow z+1), b:(z \rightarrow z+\tau)$. We define a trivial bundle $E$ over $\Sigma_{\tau}$ by two commuting matrices

$$
s(z+1)=\rho_{a} s(z), \quad s(z+\tau)=\rho_{b} s(z), \quad\left[\rho_{a}, \rho_{b}\right]=\mathrm{Id} .
$$

It is a $\operatorname{PGL}(N, \mathbb{C})$-bundle that can be lifted to $\operatorname{SL}(N, \mathbb{C})$ bundles.

Consider a representation of $\hat{\rho}$ of $\mathcal{K}$ acting on the sections of $E$ as

$$
s(z+1)=\hat{\rho}_{a} s(z), \quad s(z+\tau)=\hat{\rho}_{b} s(z) .
$$

with commutation relation $(2.4)$

$$
\left[\hat{\rho}_{a}, \hat{\rho}_{b}\right]=\omega \mathrm{Id}
$$

One can choose

$$
\hat{\rho}_{a}=\mathcal{Q}, \quad \hat{\rho}_{b}=\Lambda, \quad \mathcal{Q}=\operatorname{diag}\left(1, \omega, \ldots, \omega^{N-1}\right), \quad \Lambda=\left(\begin{array}{cccc}
0 & 1 & \ldots & 0 \\
0 & 0 & 1 & 0 \\
\vdots & \vdots & \ddots & 1 \\
1 & 0 & \ldots & 0
\end{array}\right) .
$$


The bundle with these transition functions cannot be lifted to $\operatorname{SL}(N, \mathbb{C})$ bundles. Replace $\hat{\rho}_{b}$ by

$$
\hat{\rho}_{b}^{\prime}=\exp \left(-\frac{2 \pi i}{N}\left(z+\frac{\tau}{2}\right)\right) \Lambda
$$

It is a $\operatorname{GL}(N, \mathbb{C})$ bundle since $\left[\hat{\rho}_{a}, \hat{\rho}_{b}^{\prime}\right]=I d$ and $\operatorname{det} \hat{\rho}_{b}^{\prime} \neq 1$. It follows from (2.9) that a section of the determinant bundle is the theta-function

$$
\vartheta(z, \tau)=q^{\frac{1}{8}} \sum_{n \in \mathbb{Z}}(-1)^{n} e^{\pi i(n(n+1) \tau+2 n z)}, \quad q=\exp 2 \pi i \tau .
$$

It has a simple pole in the fundamental domain $\mathbb{C} /(\mathbb{Z} \oplus \tau \mathbb{Z})$. Therefore, the bundle has degree one. It is called the theta-bundle.

To consider a general case [10] represent the rank as the product $N=p l$. Define the transition matrix

$$
\begin{aligned}
& \hat{\rho}_{a}=\mathcal{Q}, \\
& \hat{\rho}_{b}=\mathbf{e}\left(\vec{u}_{l}\right) \Lambda^{p},
\end{aligned}
$$

where

$$
\vec{u}_{l}=\operatorname{diag}(\overbrace{\mathbf{u}_{p}, \ldots, \mathbf{u}_{p}}^{l}), \quad \mathbf{u}_{p}=\left(\tilde{u}_{1}, \ldots, \tilde{u}_{p}\right) .
$$

Since $\left[\mathcal{Q}, \Lambda^{p}\right]=\omega^{p} \operatorname{Id}_{l}, \omega^{p}=\exp \frac{2 \pi i}{l}$

$$
\left[\hat{\rho}_{a}, \hat{\rho}_{b}\right]=\omega^{p} \operatorname{Id}_{N}
$$

Comparing this relation with (2.5) we conclude that (2.11) and (2.12) serve as the transition matrices for a $G_{l}$-bundle over $\Sigma_{\tau}$. Therefore $\omega^{p}$ represents an element from $H^{2}\left(\Sigma_{\tau}, \mathcal{Z}\left({ }^{L} G_{l}\right)\right) \sim \mathbb{Z}_{p}$. It is an obstruction (2.6).

As in (2.9), modify the transition matrix

$$
\hat{\rho}_{b} \rightarrow \hat{\rho}_{b}^{\prime}=\exp \left\{-\frac{2 \pi i}{p}\left(z+\frac{\tau}{2}\right)\right\} \hat{\rho}_{b} .
$$

We come to the $\operatorname{GL}(N, \mathbb{C})$-bundle of degree $p(\bmod N)$.

\subsection{Local description}

There exists another description of a holomorphic bundles over $\Sigma_{g}$. Let $w_{0}$ be a fixed point on $\Sigma_{g}$ and $D_{w_{0}}\left(D_{w_{0}}^{\times}\right)$be a disc (punctured disc) with a center $w_{0}$ with a local coordinate $z$. A bundle $E$ over $\Sigma_{g}$ can be trivialized over $D$ and over $\Sigma_{g} \backslash w_{0}$. These two trivializations are related by a $\mathrm{GL}(N, \mathbb{C})$ transformation $g(z)$, holomorphic on $D_{w_{0}}^{\times}$. If we consider another trivialization over $D$ then $g$ is multiplied from left by an invertible matrix $h$ on $D$. Likewise, a trivialization over $\Sigma_{g} \backslash w_{0}$ is determined up to the multiplication on the right $g \rightarrow g h$, where $h \in \operatorname{GL}(N, \mathbb{C})$ is holomorphic on $\Sigma_{g} \backslash w_{0}$. Thus, the set of isomorphism classes of rank $N$ vector bundles is described as a double-coset

$$
\operatorname{GL}(N, \mathbb{C})\left(D_{w_{0}}\right) \backslash \mathrm{GL}(N, \mathbb{C})\left(D_{w_{0}}^{\times}\right) / \mathrm{GL}(N, \mathbb{C})\left(\Sigma_{g} \backslash w_{0}\right),
$$

where $\operatorname{GL}(N, \mathbb{C})(U)$ denote the group of $\operatorname{GL}(N, \mathbb{C})$-valued holomorphic functions on $U$.

Let det $g(z)=1$. If $g\left(z e^{2 \pi i}\right)=g(z)$ then it defines a $\operatorname{SL}(N, \mathbb{C})$-bundle over $\Sigma_{g}$. But if the monodromy is nontrivial

$$
g\left(z e^{2 \pi i}\right)=\omega g(z), \quad \omega^{N}=1,
$$


then $g(w)$ is a transition matrix for a $\operatorname{PSL}(N, \mathbb{C})$-bundle but not for a $\operatorname{SL}(N, \mathbb{C})$-bundle. This relation is similar to $(2.4)$.

Let us choose a trivialization of $E$ over $D$ by choosing $N$ linear independent holomorphic sections $\vec{s}=\left(s_{1}, s_{2}, \ldots, s_{N}\right)$. Thereby, the bundle $E$ over $D$ is represented by a sum of $N$ line bundles $\mathcal{L}_{1} \oplus \mathcal{L}_{2} \oplus \cdots \oplus \mathcal{L}_{N}$. The sections over $\Sigma_{g} \backslash w_{0}$ are obtained by the action of the transition matrix $\vec{s}^{\prime}=\vec{s} g$.

Let $\vec{m}$ belongs to the root lattice $\left(\vec{m}=\left(m_{1}, m_{2}, \ldots, m_{N}\right) \in Q\right)$ (A.2). Transform the restriction of the section $\vec{s}$ on $D_{w_{0}}^{\times}$as

$$
s_{j} \rightarrow z^{-m_{j}} s_{j}, \quad j=1, \ldots, N .
$$

Then the transition matrix is transformed by the diagonal matrix

$$
g(z) \rightarrow \operatorname{diag}\left(z^{-m_{1}}, z^{-m_{2}}, \ldots, z^{-m_{N}}\right) g(z) .
$$

It implies the transformation of line bundles over $D$

$$
\mathcal{L}_{j} \rightarrow \mathcal{L}_{j} \otimes \mathcal{O}\left(m_{j}\right)
$$

In this way we come to the new bundle $\tilde{E}$ (the modified bundle). It is defined by the new transition matrix (2.15). This transformation of the bundle $E$ to $\tilde{E}$ (or more exactly the map of sheaves of its sections)

$$
\Gamma(E) \stackrel{\Xi(\vec{m})}{\longrightarrow} \Gamma(\tilde{E}), \quad \Xi(\vec{m}) \sim \operatorname{diag}\left(z^{-m_{1}}, z^{-m_{2}}, \ldots, z^{-m_{N}}\right),
$$

is called the modification or the Hecke transformation of type $\vec{m}=\left(m_{1}, m_{2}, \ldots, m_{N}\right)$. In fieldtheoretical terms it corresponds to the t'Hooft operator, generating by monopoles (see below).

Let us relax the condition $\sum_{j=1}^{N} m_{j}=0$. Then the modification $\Xi(\vec{m})$ changes the topology of $E$. We come to a nontrivial bundle of degree $\operatorname{deg}(\tilde{E})=\operatorname{deg}(E)+\sum_{j=1}^{N} m_{j}$. In next section we illustrate this fact.

Now assume that $\vec{m}$ belongs to the weight lattice $P$ (A.4). Then the modification $\Xi(\vec{m})$ changes the characteristic class of a $\operatorname{PGL}(N, \mathbb{C})$-bundle $E$. To prove it let us pass to the basis of the fundamental weights (A.3)

$$
\vec{m}=\sum_{j=1}^{N} m_{j} e_{j}=\sum_{k=1}^{N-1} n_{k} \varpi_{k} .
$$

It follows from (A.3) that $m_{j}$ and $n_{k}$ are related as

$$
\begin{aligned}
& m_{1}=\frac{1}{N}\left((N-1) n_{1}+(N-2) n_{2}+\cdots+n_{N-1}\right), \\
& m_{2}=\frac{1}{N}\left(-n_{1}+(N-2) n_{2}+\cdots+n_{N-1}\right), \\
& m_{N}=\frac{1}{N}\left(-n_{1}-2 n_{2}-\cdots-(N-1) n_{N-1}\right), \quad n_{k}=m_{k}-m_{k+1} .
\end{aligned}
$$

Rewrite the modification in the form of the product of the diagonal matrices

$$
\Xi(\vec{n}) \sim \prod_{k=1}^{N-1} \operatorname{diag}\left(z^{-n_{k} \varpi_{k}}\right) .
$$


It follows from (A.3) that the monodromy of this matrix around the point $z=0$ is

$$
\exp \left(-\frac{2 \pi i}{N} \sum_{k=1}^{N-1} k n_{k}\right) \operatorname{Id}_{N}
$$

Therefore, the characteristic class of the adjoint bundle is unchanged if

$$
\sum_{k=1}^{N-1} k n_{k}=N \sum_{j=1}^{N-1} m_{j}=0, \quad(\bmod N)
$$

In this case the weight vector $\vec{m}$ belongs to the root lattice $Q$. Otherwise, we come to the non-trivial monodromy (2.17). It is an obstruction to lift the $\operatorname{PGL}(N, \mathbb{C})$-bundle to a $\operatorname{SL}(N, \mathbb{C})$ bundle. This element can be identified with the monodromy (2.4) and in this way with an element from $H^{2}\left(\Sigma, \mathbb{Z}_{N}\right)$. As it was mentioned above, the modified bundle $\tilde{E}$ can be lifted to a $\operatorname{GL}(N, \mathbb{C})$ bundle. Let us act on the modified sections $(2.14)$ by the scalar matrix

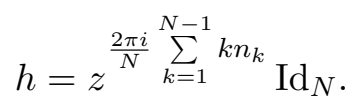

It is a $\mathrm{GL}(N, \mathbb{C})$ gauge transformation. The monodromy of the new transition matrix is trivial. Therefore, we come to the $\operatorname{GL}(N, \mathbb{C})$ bundle. The bundle is topologically nontrivial - it has degree

$$
p=\sum_{k=1}^{N-1} k n_{k}=N \sum_{j=1}^{N-1} m_{j}
$$

It follows from (2.17) that the characteristic class $\xi$ and the degree $p$ are related as

$$
\xi=\exp \frac{2 \pi i}{N} p
$$

The set of modifications that changes the degree on $p$ is defined as solutions of (2.18) in integers $n_{k}$.

Assume that the bundle $E$ is equipped with a holomorphic connection. On $D_{w_{0}}^{\times}$it takes the form $\left(\partial_{z}+A_{z}\right) d z$ and can be considered as an element of the affine Lie coalgebra $\widehat{g l}^{*}(N, \mathbb{C})\left(D_{w_{0}}^{\times}\right)$ The gauge transformation (2.15) acts on $A_{w}$ acts as the coadjoint action

$$
\left(A_{z}\right)_{j k} d z \rightarrow\left(z^{m_{k}-m_{j}}\left(A_{z}\right)_{j k}\left(1-\delta_{j k}\right)-m_{j} z^{-1} \delta_{j k}\right) d z .
$$

Let $\vec{m} \in P$. Then the first term in the r.h.s. is well defined, since $m_{k}-m_{j}$ is integer. The last term represents the shift action (A.14) of the affine group $\bar{W}_{a}$ (A.13) on the connection. The topology of $E$ is not changed if $\vec{m} \in Q$ and we come to description of the characteristic class as elements from factor group $\bar{W}_{a} / W_{a}$ (A.15). We come again to this point in Section 4.

Let $N=p l$ with $l \neq 1, N$ and $G_{l}=\operatorname{SL}(N, \mathbb{C}) / \mathbb{Z}_{l}$ (A.9). Consider the gauge transformation (2.16) with $\vec{m}(\vec{\varpi}) \in \Gamma\left({ }^{L} G\right)$ (A.9). For example, we can take $\vec{\varpi}=(p, 0, \ldots, 0)$. Then the monodromy (2.17) belongs to the group $\mathbb{Z}_{l}$. It means that the modified bundle $\tilde{E}$ is the $G_{l}$-bundle that cannot be lifted to the $\operatorname{SL}(N, \mathbb{C})$-bundle (see $(2.6)$ ).

The modification can be performed in an arbitrary number of points $w_{a},(a=1, \ldots, n)$. To this end define the isomorphism classes of vector bundles as the quotient

$$
\prod_{a=1}^{n} \operatorname{GL}(N, \mathbb{C})\left(D_{w_{a}}\right) \backslash \prod_{a=1}^{n} \operatorname{GL}(N, \mathbb{C})\left(D_{w_{a}}^{\times}\right) / \operatorname{GL}(N, \mathbb{C})\left(\Sigma_{g} \backslash\left(w_{1}, \ldots, w_{a}\right)\right) .
$$


We have $n$ transition matrices $g_{a}\left(z_{a}\right)$ representing an element of the quotient, where $z_{a}$ is a local coordinate. Let $\Xi\left(\vec{m}_{a}\right)$ denotes the modification of $E$ at $w_{a}$ and $\Xi=\prod_{a=1}^{n} \Xi\left(\vec{m}_{a}\right)$. The order of modifications in the product is irrelevant, since they commute. To calculate the monodromy of $\Xi$ we choose the same orientation in all points $w_{a}$. The characteristic class of $\xi$ of modified bundle $\tilde{E}$ corresponds to

$$
\prod_{a=1}^{n} \exp \left(-\frac{2 \pi i}{N} \sum_{k=1}^{N-1} k n_{k}^{a}\right) \text {. }
$$

\section{Bogomolny equation}

Definition. Let $W=\mathbb{R} \times \Sigma_{g}$. Consider a bundle $V$ over $W$ equipped with the curvature $F$. Let $\phi$ be a zero form on $W$ taking value in sections of the adjoint bundle $\phi \in \Omega^{0}(W$, End $V)$. It is the so-called Higgs field.

The Bogomolny equation on $W$ takes the form

$$
F=* D \phi .
$$

Here $*$ is the Hodge operator on $W$ with respect to the metric $d s^{2}$ on $W$. In local coordinates $(z, \bar{z})$ on $\Sigma_{g}$ and $y$ on the real line $d s^{2}=g|d z|^{2}+d y^{2}$, where $g(z, \bar{z})|d z|^{2}$ is a metric on $\Sigma_{g}$. Then the Hodge operator is defined as

$$
\star d y=\frac{1}{2} i g d z \wedge d \bar{z}, \quad \star d z=-i d z \wedge d y, \quad \star d \bar{z}=i d \bar{z} \wedge d y,
$$

and (3.1) becomes

$$
\begin{aligned}
\partial_{z} A_{\bar{z}}-\partial_{\bar{z}} A_{z}+\left[A_{z}, A_{\bar{z}}\right] & =\frac{i g(z, \bar{z})}{2}\left(\partial_{y} \phi+\left[A_{y}, \phi\right]\right), \\
\partial_{y} A_{z}-\partial_{z} A_{y}+\left[A_{y}, A_{z}\right] & =i\left(\partial_{z} \phi+\left[A_{z}, \phi\right]\right), \\
\partial_{y} A_{\bar{z}}-\partial_{\bar{z}} A_{y}+\left[A_{y}, A_{\bar{z}}\right] & =-i\left(\partial_{\bar{z}} \phi+\left[A_{\bar{z}}, \phi\right]\right) .
\end{aligned}
$$

In what follows we will consider only $\operatorname{PSL}(N, \mathbb{C})$-bundles.

A monopole solution of this equation is defined in the following way. Let $\tilde{W}=\left(W \backslash \vec{x}^{0}=\right.$ $\left.\left(y=0, z=z_{0}\right)\right)$. The Bianchi identity $D F=0$ on $\tilde{W}$ implies that $\phi$ can be identified with the Green function for the operator $\star D \star D$

$$
\begin{aligned}
& \star D \star D \phi=M \delta\left(\vec{x}-\vec{x}^{0}\right), \\
& M=\operatorname{diag}\left(m_{1}, m_{2}, \ldots, m_{N}\right) \in \operatorname{gl}(N, \mathbb{C}), \quad \vec{m}=\left(m_{1}, m_{2}, \ldots, m_{N}\right) \in P \quad(\text { A.4 }),
\end{aligned}
$$

and $\left(m_{1}, m_{2}, \ldots, m_{N}\right)$ are the monopole charges. We explain below this choice of $M$. This equation means that $\phi$ is singular at $\vec{x}^{0}$.

Boundary conditions and gauge symmetry. In what follows except Section 3.1 we assume that $\partial_{y} \phi$ vanishes when $y \rightarrow \pm \infty$. It is the Neumann boundary conditions for the Higgs field, while the gauge fields are unspecified. Let $V_{ \pm}$be restrictions of $V$ to the bundles over $\Sigma_{g}$ on the "left end" and "right end" of $W: y \rightarrow \pm \infty$. These bundles are flat. It follows from (3.7a), where the gauge $A_{y}=0$ is assumed. It was proved in [9] that in absence of the source $M=0$ in (3.3) the only solutions of (3.1) with these boundary conditions are $F=0, \phi=0$. Note that these boundary conditions differ from ones chosen in [9].

The Bogomolny equation defines a transformation $V_{-} \rightarrow V_{+}$. ( $E$ and $\tilde{E}$ in our notations in Introduction.) We will see in next sections that in general the characteristic classes of bundles are changed under these transformations. It depends on the monopole charges $\vec{m}=\left(m_{1}, m_{2}, \ldots, m_{N}\right)$. 
The system (3.2) is invariant with respect to the gauge group $\mathcal{G}$ action:

$$
\begin{aligned}
A_{z} \rightarrow h A_{z} h^{-1}+\partial_{z} h h^{-1}, & A_{\bar{z}} \rightarrow h A_{\bar{z}} h^{-1}+\partial_{\bar{z}} h h^{-1}, \\
A_{y} \rightarrow h A_{y} h^{-1}+\partial_{y} h h^{-1}, & \phi \rightarrow h \phi h^{-1},
\end{aligned}
$$

where $h \in \mathcal{G}$ is a smooth map $W \rightarrow \mathrm{GL}(N, \mathbb{C})$. To preserve the r.h.s. in (3.3) it should satisfy the condition $\left[h\left(\vec{x}^{0}\right), M\right]=0$.

Assume for simplicity that $V$ is an adjoint bundle. Since the gauge fields for $y= \pm \infty$ are unspecified and only flat we can act on them by boundary values of the gauge group $\left.\mathcal{G}\right|_{y= \pm \infty}=\mathcal{G}_{ \pm}$. Then $\mathcal{M}_{ \pm}=\left\{V_{ \pm}\right\} / \mathcal{G}_{ \pm}$are the moduli spaces of flat bundles.

Relations to integrable systems. The moduli spaces of flat bundles are phase spaces of non-autonomous Hamiltonian systems related to the isomonodromy problems over $\Sigma_{g}$. The isomonodromy problem takes the form

$$
\left[\partial_{z}+A_{z}, \Psi\right]=0, \quad\left[\partial_{\bar{z}}+A_{\bar{z}}, \Psi\right]=0 .
$$

Here $\Psi \in \Omega^{0}\left(\Sigma_{g}\right.$, Aut $\left.V\right)$ is the Baker-Akhiezer function. These system is compatible for any degree of bundle, because it is defined in the adjoint representation. One example of these systems we have mentioned in Introduction $\left(V_{-} \rightarrow\right.$ Painlevé VI) and ( $V_{+} \rightarrow$ Zhukovsky-Volterra gyrostat).

It is known, that the moduli space of flat bundles are deformation (the Whitham deformation) of the phase spaces of the Hitchin integrable systems - the moduli spaces of the Higgs bundles. To consider this limit one should replace a holomorphic connection by the $\kappa$-connection $\kappa \partial_{z}+A_{z}$ introduced by P. Deligne and take a limit $\kappa \rightarrow 0$. It is a quasi-classical limit in the linear problem (3.6). Details can be found in $[11,12,13,14]$. In this way a monopole solution put in a correspondence (symplectic Hecke correspondence) two Hitchin systems (the first and the last examples in Introduction). But Bogomolny equation tells us more. It describes an evolution from one type of system to another.

It is possible to generalize (3.3) and consider multi-monopole sources $\sum_{a} M_{a} \delta\left(\vec{x}-\vec{x}_{a}^{0}\right)$ in the r.h.s. This generalization will correspond to modifications in a few points of $\Sigma_{g}$ described at the end of previous section.

It is interesting that in some particular cases this situation was discussed in the frameworks of a supersymmetric Yang-Mills theory $[15,16]^{1}$. It was observed there that a monopole configuration corresponds to a soliton type evolution along $y$. Therefore, it can be suggested that the system (3.2) is integrable. We did not succeed to prove this fact, but propose a linear problem related to the Bogomolny equation. An associated linear problem allows one in principal to apply the methods of the Inverse Scattering Problem or the Whitham approximation to find solutions [18]. Assume that the metric $g$ on $\Sigma_{g}$ is a constant. Then the system (3.2) is the compatibility condition for the linear system

$$
\begin{aligned}
& \left(\partial_{z}+A_{z}+\frac{1}{2} \lambda^{-1} g\left(\partial_{y}+A_{y}+i \phi\right)\right) \psi=0, \\
& \left(\partial_{\bar{z}}+A_{\bar{z}}+\frac{1}{2} \lambda g\left(\partial_{y}+A_{y}-i \phi\right)\right) \psi=0,
\end{aligned}
$$

where $\lambda \in \mathbb{C} P^{1}$ is a spectral parameter. It can be suggested that monopole solution of (3.2) corresponds to a soliton solution of this system. We will not develop here this approach ${ }^{2}$.

Gauge fixing. Choose a gauge fixing conditions as: $A_{\bar{z}}=0$. Holomorphic functions $h=h(y, z)$ preserve this gauge. Then

$$
-\partial_{\bar{z}} A_{z}=\frac{i g}{2}\left(\partial_{y} \phi+\left[A_{y}, \phi\right]\right)
$$

\footnotetext{
${ }^{1}$ We are grateful to A. Gorsky who bring our attention to this point.

${ }^{2}$ The $\mathrm{SU}(2)$ case and $W=\mathbb{R}^{3}$ was analyzed in [19] for different boundary conditions.
} 


$$
\begin{aligned}
& \partial_{y} A_{z}-\partial_{z} A_{y}+\left[A_{z}, A_{y}\right]=i\left(\partial_{z} \phi+\left[A_{z}, \phi\right]\right), \\
& \partial_{\bar{z}} A_{y}=i \partial_{\bar{z}} \phi .
\end{aligned}
$$

The last equation means that $A_{y}-i \phi$ is holomorphic. It follows from (3.5) that the gauge transformation of this function is

$$
A_{y}-i \phi \rightarrow h\left(A_{y}-i \phi\right) h^{-1}+\partial_{y} h h^{-1} .
$$

Thus, we can keep $A_{y}=i \phi$ by using holomorphic and $y$-independent part of the gauge group $\left(\partial_{y} h=0\right)$. Finally, we come to the system

$$
\begin{aligned}
& \partial_{\bar{z}} A_{z}=-\frac{i g}{2} \partial_{y} \phi \\
& \partial_{y} A_{z}-2 i \partial_{z} \phi+2 i\left[A_{z}, \phi\right]=0, \\
& A_{y}=i \phi \\
& A_{\bar{z}}=0 .
\end{aligned}
$$

Two upper equations from (3.7) lead to the Laplace type equation

$$
\partial_{y}^{2} \phi+\frac{4}{g}\left(\partial_{z} \partial_{\bar{z}} \phi+\partial_{\bar{z}}\left[A_{z}, \phi\right]\right)=0
$$

In scalar case (3.8) is simplified

$$
\partial_{y}^{2} \phi+\frac{4}{g} \partial_{z} \partial_{\bar{z}} \phi=0
$$

\subsection{Rational solution in scalar case}

In this subsection we replace $\Sigma_{g}$ by $\mathbb{C}$. The coordinates $z, \bar{z}$ on $\mathbb{C}$ will play the role of local coordinates on $\Sigma_{g}$. Consider (3.9) on $\tilde{W}=\mathbb{R} \times \mathbb{C} \backslash(0,0,0)$. In this particular case we can choose the boundary conditions in the following form:

$$
\begin{aligned}
& \left.\phi\right|_{y= \pm \infty}=0, \\
& \left.A_{z}\right|_{y= \pm \infty}=0 .
\end{aligned}
$$

The solution of (3.9) with $g=1$ satisfying (3.10) has the form:

$$
\phi=c \frac{1}{\sqrt{y^{2}+z \bar{z}}}
$$

where $c$ is a constant. So in fact we deal here with the Laplace equation on $\mathbb{R} \times \mathbb{C} \backslash(0,0,0)$. It follows from (3.11) and from the equation $\partial_{\bar{z}} A_{z}=-\frac{i}{2} \partial_{y} \phi(3.7 \mathrm{a})$ that

$$
\begin{array}{ll}
A_{z}(z, \bar{z}, y)=A_{z}^{+}(z, \bar{z}, y), & y>0 \quad \text { and } \quad y=0, \quad z \neq 0, \\
A_{z}(z, \bar{z}, y)=A_{z}^{-}(z, \bar{z}, y), \quad & y<0,
\end{array}
$$

where

$$
\begin{aligned}
& A_{z}^{+}(z, \bar{z}, y)=-i c\left(\frac{1}{z} \frac{y}{\sqrt{y^{2}+z \bar{z}}}-\frac{1}{z}\right)+\text { const } \\
& A_{z}^{-}(z, \bar{z}, y)=-i c\left(\frac{1}{z} \frac{y}{\sqrt{y^{2}+z \bar{z}}}+\frac{1}{z}\right)+\text { const }
\end{aligned}
$$


and $A_{z}(z, \bar{z}, y)$ is a connection on the line bundle $\mathcal{L}$ over $\tilde{W}$. The connection has a jump $-2 i c \frac{1}{z}$ at $y=0$. To deal with smooth connections we compensate it by a holomorphic gauge transform that locally near $\vec{x}_{0}$ has the form $h \sim z^{m}$. Here $m$ should be integer, because $h$ is a smooth function. Notice that all holomorphic line bundles over $S^{2}$ are known to be $\mathcal{O}(m)$-bundles, $m \in \mathbb{Z}$. Thus, we have $c=i \frac{m}{2}, m \in \mathbb{Z}$. This usually referred as a quantization of the monopole charge. In fact the constant $c$ contains factor $4 \pi$ (area of a unit sphere) which yields a proper normalization of delta-function and appears in Gauss's law. The gauge transformation $h$ is the modification $(2.14),(2.15)$ for line bundles over $\mathbb{C} P^{1}$. This is what we mean saying that the described 3-dimensional construction characterizes the modification of the corresponding bundle.

Consider for a moment the general situation $W=\mathbb{R} \times \Sigma_{g}$ and let $z, \bar{z}$ be local coordinates on $\Sigma_{g}$. Locally near $\vec{x}_{0}=(0,0,0)$ connections corresponding to solutions of (3.9) have the form (3.13). Let $S^{2}$ be a small sphere surrounding the point $\vec{x}_{0}$ in $W$ and $\Sigma_{g, \pm}$ be the left and right boundaries of $W$ and $\mathcal{L}_{ \pm}$are the corresponding restrictions of $\mathcal{L}$. Then (as it is explained in [9] in detail)

$$
\int_{\Sigma_{g,+}} F=\int_{\Sigma_{g,-}} F+m
$$

where $F$ is a curvature of the connection $A$. In other words, the monopole solution with the charge $m$ increases the degree of bundle by $m\left(\operatorname{deg} \mathcal{L}_{+}=\operatorname{deg} \mathcal{L}_{-}+m\right)$.

\subsection{Elliptic solution in scalar case}

The Laplace equation (3.9) on $\Sigma_{\tau}$ has the form

$$
\partial_{y}^{2} \phi+4(\mathcal{I} m(\tau))^{2} \partial_{z} \partial_{\bar{z}} \phi=0
$$

or

$$
\partial_{y}^{2} \phi+(2 \pi \alpha)^{2} \partial_{z} \partial_{\bar{z}} \phi=0, \quad \alpha^{-1}=\frac{2 \pi i}{\tau-\bar{\tau}}
$$

and $\operatorname{Im}(\tau)$ is the area of parallelogram of periods. We give two representations of the Green function $\phi$ and prove their equivalence using the same technique as for the Kronecker series described in [17].

A naive elliptic solution of (3.14) on $\tilde{W}$ is obtained by averaging (3.12) over the lattice $\Gamma=\mathbb{Z} \oplus \tau \mathbb{Z} \subset \mathbb{C}:^{3}$

$$
\phi(z, y)=c \sum_{\gamma \in \Gamma} \frac{1}{\sqrt{(\pi \alpha y)^{2}+|z+\gamma|^{2}}} .
$$

However the series diverges. That is why we consider its generalization

$$
\mathcal{R}(s, x, z, y)=c \sum_{\gamma \in \Gamma} \frac{\chi(\gamma, x)}{\left((\pi \alpha y)^{2}+|z+\gamma|^{2}\right)^{s}}, \quad \mathcal{R}\left(\frac{1}{2}, 0, z, y\right)=\phi(z, y),
$$

where

$$
\chi(\gamma, x)=e^{\alpha^{-1}(\gamma \bar{x}-\bar{\gamma} x)}
$$

\footnotetext{
${ }^{3}$ We omit here and in what follows the $\bar{z}$ dependence.
} 
is a character $\mathbb{Z} \times \mathbb{Z} \rightarrow \mathbb{C}^{*}$ of the additive group $\Gamma$ and $s, x$ are complex parameters. The characters are double-periodic

$$
\chi(\gamma, x+1)=\chi(\gamma, x), \quad \chi(\gamma, x+\tau)=\chi(\gamma, x), \quad \gamma \in \Gamma,
$$

while the series $\mathcal{R}(s, x, z, y)$ are quasi-periodic

$$
\begin{aligned}
& \mathcal{R}(s, x, z+1, y)=e^{\alpha^{-1}(x-\bar{x})} \mathcal{R}(s, x, z, y), \\
& \mathcal{R}(s, x, z+\tau, y)=e^{\alpha^{-1}(x \bar{\tau}-\bar{x} \tau)} \mathcal{R}(s, x, z, y) .
\end{aligned}
$$

The variable $x$ describes behavior of $\mathcal{R}(s, x, z, y)$ on the lattice $\Gamma$. In other words, $x$ parameterizes the moduli space of line bundles on $\Sigma_{\tau}$. Note that for $\mathcal{R} e s>1$ the series in the r.h.s. of (3.16) converges. The function

$$
\mathcal{R}\left(\frac{1}{2}, x, z, y\right)=c \sum_{\gamma \in \Gamma} \frac{\chi(\gamma, x)}{\left((\pi \alpha y)^{2}+|z+\gamma|^{2}\right)^{\frac{1}{2}}}=\phi(x, z, y)
$$

is the formal solution of (3.14) with the quasi-periodicity conditions (3.17).

Another representation of the Green function can be obtained by the Fourier transform. Define the delta-functions

$$
\delta(y)=\int_{-\infty}^{+\infty} d p e^{2 \pi i p y}, \quad \delta^{(2)}(z, \bar{z})=\sum_{\gamma \in \Gamma} \chi(\gamma, z) .
$$

Then

$$
\sum_{\gamma \in \Gamma} \chi(\gamma+x, z)=\chi(x, z) \sum_{\gamma \in \Gamma} \chi(\gamma, z)=\chi(x, z) \delta^{2}(z, \bar{z})=\delta^{2}(z, \bar{z}),
$$

Let $\tilde{\phi}$ be the Green function with the quasi-periodicity (3.17)

$$
\partial_{y}^{2} \tilde{\phi}+(2 \pi \alpha)^{2} \partial_{z} \partial_{\bar{z}} \tilde{\phi}=c \delta(y) \delta^{(2)}(z, \bar{z}) .
$$

Expanding it in the Fourier harmonics we find

$$
\tilde{\phi}=-\frac{c}{4 \pi^{2}} \sum_{\gamma \in \Gamma} \int_{-\infty}^{+\infty} d p \frac{e^{2 \pi i p y}}{p^{2}+|\gamma+z|^{2}} \chi(\gamma+x, z) .
$$

Integrating over $p$ provides factor $\pi$ and leads to the following expression:

$$
\tilde{\phi}(x, z, y)=-\frac{c}{4 \pi} \sum_{\gamma \in \Gamma} \frac{1}{|\gamma+x|} e^{-2 \pi|\gamma+x||y|} \chi(\gamma+x, z) .
$$

It is worthwhile to note that the solution (3.19) is well defined. Our goal is to find interrelations between (3.19) and (3.18).

Consider a generalization of $(3.19)$

$$
I(s, x, z, y)=2 c \pi^{s} y^{s-\frac{1}{2}} \sum_{\gamma \in \Gamma} \frac{K_{s-\frac{1}{2}}(2 \pi|y||\gamma+x|)}{|\gamma+x|^{s-\frac{1}{2}}} \chi(\gamma+x, z) .
$$


Here $K_{\nu}$ is the Bessel-Macdonald function

$$
K_{\nu}(2 \pi y z)=\frac{\Gamma\left(\nu+\frac{1}{2}\right)(z)^{\nu}}{2(\pi y)^{\nu} \Gamma\left(\frac{1}{2}\right)} \int_{-\infty}^{+\infty} d p \frac{e^{2 \pi i p y}}{\left(p^{2}+z^{2}\right)^{\nu+\frac{1}{2}}} .
$$

The function $I(s, x, z, y)$ is the Green function for the pseudo-differential operator

$$
\left(\partial_{y}^{2}+4 \alpha^{2} \pi^{2} \partial_{z} \partial_{\bar{z}}\right)^{s}
$$

on $\mathbb{R} \times \Sigma_{\tau}$ with the boundary conditions (3.17). Since

$$
K_{\frac{1}{2}}(x)=\sqrt{\frac{\pi}{2 x}} e^{-x}
$$

we conclude that for $s=1 I$ coincides with $\tilde{\phi}(3.19)$ up to constant.

We are going to establish a relation between (3.16) and (3.20), and in this way between (3.15) and (3.19). Let us prove that

$$
I(s, x, z, y)=c \sum_{\gamma \in \Gamma} \int_{-\infty}^{\infty} d p \int_{0}^{\infty} \frac{d t}{t} t^{s} e^{-t\left(p^{2}+|\gamma+x|^{2}\right)+2 \pi i p y} \chi(\gamma+x, z) .
$$

In fact, using the integral representation for the Gamma-function

$$
\Gamma(s)=\int_{0}^{\infty} \frac{d t}{t} t^{s} e^{-t}
$$

and taking the integral over $t$ in (3.21) we come to (3.20).

The representation (3.21) is universal and can serve to define $\mathcal{R}(3.16)$

Lemma 3.1. The function $\mathcal{R}(s, x, z, y)$ has a representation as the Fourier integral

$$
\mathcal{R}(s, x, z, y)=\frac{1}{\Gamma(s) \chi(\gamma, z)} \int_{\infty}^{\infty} d k I\left(s, z, x, \frac{k}{\pi \alpha}\right) e^{-2 \pi i k y} .
$$

Proof. Substitute in (3.23) $I(s, x, z, y)(3.21)$ and take first integral over $k$. We come to the condition $p=\pi \alpha y$. Then using the integral representation for the Gamma-function (3.22) we obtain (3.16).

Remark 3.1. The series (3.20) is a three-dimensional generalization of the Kronecker series (see [17])

$$
K\left(x, x_{0}, s\right)=\sum_{\gamma} \chi\left(\gamma, x_{0}\right)|x+\gamma|^{-2 s} .
$$

Using the Poisson summation formula Kronecker proved that

$$
\Gamma(s) K\left(x, x_{0}, s\right)=\alpha^{1-2 s} \Gamma(1-s) K\left(x_{0}, x, 1-s\right) \chi\left(x, x_{0}\right) .
$$

Our purpose is to generalize this functional equation for the 3 -dimensional case $\Sigma_{\tau} \times \mathbb{R}$. It takes the following form. 
Lemma 3.2. The function $I(s, x, z, y)$ satisfies the functional equation:

$$
I(s, x, z, y)=\chi(x, z) \pi^{-\frac{1}{2}} \alpha^{-2 s+1} \int_{-\infty}^{+\infty} d k I\left(\frac{3}{2}-s, z, x, \frac{k}{\pi \alpha}\right) e^{-2 \pi i k y} .
$$

Proof. Following [17] we subdivide integral (3.21) into two parts

$$
\begin{aligned}
I(s, x, z, y)= & c \sum_{\gamma \in \Gamma} \int_{-\infty}^{\infty} d p \int_{0}^{T} \frac{d t}{t} t^{s} e^{-t\left(p^{2}+|\gamma+x|^{2}\right)+2 \pi i p y} \chi(\gamma+x, z) \\
& +c \sum_{\gamma \in \Gamma} \int_{-\infty}^{\infty} d p \int_{T}^{\infty} \frac{d t}{t} t^{s} e^{-t\left(p^{2}+|\gamma+x|^{2}\right)+2 \pi i p y} \chi(\gamma+x, z), \quad T \in \mathbb{R}, \quad T>0 .
\end{aligned}
$$

The second term is a well defined function for all $s$. Consider the first one. It is well known that for the series

$$
\Theta\left(t, x, x_{0}\right)=\sum_{\gamma} e^{-t|x+\gamma|^{2}} \chi\left(\gamma, x_{0}\right)
$$

the following functional equation holds:

$$
\Theta\left(t, x, x_{0}\right)=(\alpha t)^{-1} \Theta\left(\alpha^{-2} t^{-1}, x_{0}, x\right) \chi\left(x_{0}, x\right) .
$$

The latter follows from the Poisson summation formula which states that the averaging of function over some lattice equals the averaging of its Fourier transform over the dual lattice. In the above case the functional equation appears after the Fourier transform for the Gauss integral. Then

$$
\begin{aligned}
& \sum_{\gamma \in \Gamma} \int_{-\infty}^{\infty} d p \int_{0}^{T} \frac{d t}{t} t^{s} e^{-t\left(p^{2}+|\gamma+x|^{2}\right)+2 \pi i p y} \chi(\gamma+x, z) \\
& =\sum_{\gamma \in \Gamma} \int_{-\infty}^{\infty} d p \int_{0}^{T} \frac{d t}{t} t^{s} e^{-t p^{2}-\alpha^{-2} t^{-1}|\gamma+z|^{2}+2 \pi i p y} \chi(\gamma+z, x) \chi(x, z)(\alpha t)^{-1} \\
& \stackrel{\text { integrating }}{\text { over }}=\sum_{\gamma \in \Gamma} \int_{0}^{T} \frac{d t}{t} t^{s} e^{-\pi^{2} y^{2} t^{-1}-\alpha^{-2} t^{-1}|\gamma+z|^{2}} \chi(\gamma+z, x) \chi(x, z)(\alpha t)^{-1} \sqrt{\frac{\pi}{t}} \\
& \text { making } \\
& \stackrel{\substack{\text { mabstitution } \\
\alpha^{-2} t^{-1}=}}{=} \sum_{\gamma \in \Gamma} \int_{\alpha^{-2} T^{-1}}^{\infty} \frac{d t}{t} t^{\frac{3}{2}-s} \sqrt{\pi} \alpha^{2-2 s} e^{-t\left((\pi \alpha y)^{2}+|\gamma+z|^{2}\right)} \chi(\gamma+z, x) \chi(x, z) \text {. }
\end{aligned}
$$

Let $T=\alpha^{-1}$. Then

$$
\begin{aligned}
I(s, x, z, y)= & c \sum_{\gamma \in \Gamma} \int_{\alpha^{-1}}^{\infty} \frac{d t}{t} t^{\frac{3}{2}-s} \sqrt{\pi} \alpha^{2-2 s} e^{-t\left((\pi \alpha y)^{2}+|\gamma+z|^{2}\right)} \chi(\gamma+z, x) \chi(x, z) \\
& +c \sum_{\gamma \in \Gamma} \int_{-\infty}^{\infty} d p \int_{\alpha^{-1}}^{\infty} \frac{d t}{t} t^{s} e^{-t\left(p^{2}+|\gamma+x|^{2}\right)+2 \pi i p y} \chi(\gamma+x, z) .
\end{aligned}
$$

The proof follows from (3.25). One should only substitute $I(s, x, z, y)$ from (3.25), into (3.24). Formula (3.25) represents $I$ as the sum of two terms. Direct evaluation shows that the first (of two) term from the l.h.s. of (3.24) equals to the second one from the r.h.s. and vice versa. 
From Lemmas 3.1 and 3.2 we come to the main result of this section

$$
\mathcal{R}\left(\frac{3}{2}-s, x, z, y\right)=\frac{\sqrt{\pi} \alpha^{2 s-1}}{\Gamma\left(\frac{3}{2}-s\right)} I(s, x, z, y) .
$$

Now put $s=1$. Then one can see that well-defined series

$$
\pi \sum_{\gamma \in \Gamma} \chi(\gamma+x, z) \frac{e^{-2 \pi|y||\gamma+x|}}{|\gamma+x|}
$$

describes the analytic continuation of the divergent series

$$
\pi \sum_{\gamma \in \Gamma} \chi(\gamma, x) \frac{1}{\sqrt{(\pi \alpha y)^{2}+|\gamma+z|^{2}}}
$$

We use (3.19) as the Green function. Then

$$
\begin{aligned}
& A_{z}(z, \bar{z}, y, x)=-\frac{i c}{4 \pi} \frac{1}{\pi^{2} \alpha^{2}} \operatorname{sgn}(y) \sum_{\gamma \in \Gamma} \frac{1}{\gamma+x} e^{-2 \pi|\gamma+x||y|} \chi(\gamma+x, z), \\
& \operatorname{sgn}(y)=1 \quad \text { for } \quad y \geq 0, \quad \operatorname{sgn}(y)=-1 \quad \text { for } \quad y<0 .
\end{aligned}
$$

Notice that the jump of $A$ (while coming through $y=0, z=0$ ) is obviously defined by the jump of $\operatorname{sgn}(y)$.

Remark 3.2. Note that (3.27) is a formal solution of the Bogomolny equation. For $x \neq 0$ it is not a connection of a line bundle over $\Sigma_{\tau}$ due to its monodromies similar to (3.17). We will use this solution in next section to define a genuine connection for higher ranks bundles.

In order to compare elliptic configuration with the rational we take $x=0$. Then on the line $y=0$ the connection is proportional to

$$
A_{z} \sim \sum_{\gamma \neq 0} \frac{1}{\gamma} \chi(\gamma, z)=E_{1}(z)-\alpha^{-1}(z-\bar{z})
$$

where $E_{1}(z)=\partial \ln \vartheta(z)$ is the so-called first Eisenstein series and $\vartheta(z)$ is the theta-function (2.10). $E_{1}(z)$ has a simple pole at $z=0$ with $\operatorname{Res}_{z=0} E_{1}(z)=1$ and the connection $A_{z}$ is double-periodic. In terms of (3.5) the gauge transformation $h$ compensating the jump of the connection is given by an integer power of theta function $\vartheta^{m}(z), m \in \mathbb{Z}$. Thus

$$
\partial \log h=\partial \log \vartheta^{m}(z)=m E_{1}(z) .
$$

\section{Arbitrary rank case}

Here we describe modification of vector bundles of an arbitrary rank. First, we repeat arguments of [9] and justify the choice $M$ in (3.4). As before, we consider $\operatorname{PSL}(N, \mathbb{C})=G_{a d}$-bundles.

Near the singular point $\vec{x}^{0}$ the bundle $V$ is splited in a sum of line bundles. Using the solution (3.12) for a line bundle we take the Higgs field near the singularity in the form

$$
\phi=\frac{i}{2 \sqrt{y^{2}+z \bar{z}}} \operatorname{diag}\left(m_{1}, \ldots, m_{N}\right) .
$$

It follows from (3.13) that $A_{z}$ undergoes a discontinuous jump at $y=0$

$$
A_{z}^{+}-A_{z}^{-}=\frac{i}{z} \operatorname{diag}\left(m_{1}, m_{2}, \ldots, m_{N}\right) .
$$


To get rid of the singularity of $A$ at $z=0$, as in the Abelian case, one can perform the singular gauge transform $\Xi$ that behaves near $z=0$ as (2.16)

$$
\Xi=\operatorname{diag}\left(z^{-m_{1}}, z^{-m_{2}}, \ldots, z^{-m_{N}}\right) .
$$

Assume that $\vec{m}$ belongs to the weight lattice $\vec{m} \in P$. It means that $\Xi$ is inverse to the cocharacter $\gamma_{a d}$ of $\operatorname{PSL}(N, \mathbb{C})\left(\gamma_{a d} \in t\left(G_{a d}\right)=P^{\vee} \sim P(\right.$ A.5) $)$. As it was explained before, the modified bundle $V_{+}$can not be lifted to a $\operatorname{SL}(N, \mathbb{C})$ bundle. On the other hand, if $\vec{m}=\left(m_{1}, \ldots, m_{N}\right)$ belongs to the root lattice $Q$ (A.5), then $\Xi^{-1}=\bar{\gamma}$ and there is no obstruction to lift $V_{+}$to an $\mathrm{SL}(N, \mathbb{C})$ bundle. Note that (4.1) describes the affine group $\bar{W}_{a}$ (A.13) action in the former case and the affine group $W_{a}$ (A.12) action in the latter case. From field-theoretical point of view it is an action of the t'Hooft operator on $A_{z}$ (see (2.19)).

If $N=p l,(l \neq 1, N)$ one can consider the intermediate situation and $\gamma_{G_{l}}$ (A.8). It means that $\vec{m} \in t\left({ }^{L} G_{l}\right) \sim \Gamma\left(G_{p}\right)$. This embedding provides the modification that allows the $\operatorname{PGL}(N, \mathbb{C})$ bundle to lift to the $G_{l}=\operatorname{SL}(N, \mathbb{C}) / \mathbb{Z}_{l}$-bundle but not to a $\operatorname{SL}(N, \mathbb{C})$ bundle. In this way the monopole charges are related to the characteristic classes of bundles.

One can use the maps to the Cartan subgroups of the solution $\phi(z)$ for a line bundle over $\Sigma_{\tau}$ (3.26) with $x=0$

$$
\phi \rightarrow \phi \cdot \operatorname{diag}\left(m_{1}, m_{2}, \ldots, m_{N}\right) .
$$

Unfortunately, in this case $V$ being restricted on $\Sigma_{\tau}$ is splitting globally over $\Sigma_{\tau}$ and defines an unstable bundle, though it allows one to describe its modifications.

There exists a map of $\phi(z, y, x)$ and $A_{z}(z, y, x)$ with $x \neq 0$ to a non-semisimple elements of $\operatorname{sl}(N, \mathbb{C})$

$$
\begin{gathered}
\left(\begin{array}{cccc}
0 & k_{1} \phi\left(z, y, x_{1}\right) & \ldots & k_{N-1} \phi\left(z, y, x_{N-1}\right) \\
0 & 0 & \ldots & 0 \\
\vdots & \ldots & \ddots & \vdots \\
0 & \ldots & \ldots & 0
\end{array}\right) \\
\left(\begin{array}{cccc}
0 & k_{1} A_{z}\left(z, y, x_{1}\right) & \ldots & k_{N-1} A_{z}\left(z, y, x_{N-1}\right) \\
0 & 0 & \ldots & 0 \\
\vdots & \ldots & \ddots & \vdots \\
0 & \ldots & \ldots & 0
\end{array}\right)
\end{gathered}
$$

Since these matrices commute they are solutions of the matrix equation (3.8). The connection has a jump at $y=0$. The bundle is characterized by the diagonal monodromy matrices (2.7)

$$
\rho_{a}=\operatorname{diag}\left(a_{1}, a_{2}, \ldots, a_{N}\right), \quad \rho_{b}=\operatorname{diag}\left(b_{1}, b_{2}, \ldots, b_{N}\right),
$$

where

$$
\begin{array}{ll}
a_{1}=\prod_{j=1}^{N-1} \sigma_{j}^{\frac{1}{N}}, & a_{2}=a_{1} \sigma_{1}^{-1}, \quad a_{N}=a_{1} \sigma_{N-1}^{-1}, \\
b_{1}=\prod_{j=1}^{N-1} \varsigma_{j}^{\frac{1}{N}}, & b_{2}=a_{1} \varsigma_{1}^{-1}, \quad b_{N}=a_{1} \varsigma_{N-1}^{-1},
\end{array}
$$

$\sigma_{j}=\exp \left(\alpha^{-1}\left(x_{j}-\bar{x}_{j}\right)\right), \varsigma_{j}=\exp \left(\alpha^{-1}\left(x_{j} \bar{\tau}-\bar{x}_{j} \tau\right)\right)$. Note that they are $y$-independent. Moreover, the singular gauge transform, leading to a continues solution of the Bogomolny equation, belongs to the upper nilpotent subgroup and in this way does not change the topological type of the bundle. 
Now we describe non-diagonal modifications $\Xi$ of a $\operatorname{PGL}(N, \mathbb{C})$-bundles over $\Sigma_{\tau}$. We do not know solutions of the Bogomolny equation in this case, and only can assert that the modification "kill the jump" of $A_{z}$ at $y=0$ :

$$
\Xi^{-1} \partial_{z} \Xi=A_{z}^{+}-\Xi^{-1} A_{z}^{-} \Xi
$$

We use the global description of a bundle $E$ in terms of the transition matrices $\rho_{a}, \rho_{b}(2.7)$ using the approach of [1]. Let

$$
\rho_{a}=\operatorname{Id}_{N}, \quad \rho_{b}=\mathbf{e}-u, \quad\left(u=\operatorname{diag}\left(u_{1}, u_{2}, \ldots, u_{N}\right)\right), \quad \mathbf{e}(a)=\exp (2 \pi i a) .
$$

The group commutator of these matrices is $\operatorname{Id}_{N}$. Thereby, $E$ can be lifted to a $\mathrm{SL}(N, \mathbb{C})$-bundle.

Define a modification $\Xi$ of $E$ to the bundle $\tilde{E}$ with the transition matrices (2.8). Then $\Xi$ should intertwine the transition matrices

$$
\begin{aligned}
& \Xi(z+1, \tau)=\mathcal{Q} \times \Xi(z, \tau), \\
& \Xi(z+\tau, \tau)=\Lambda(z, \tau) \times \Xi(z, \tau) \times \operatorname{diag}(\mathbf{e}(u))
\end{aligned}
$$

The matrix $\Xi(z)$ degenerates at $z=0$ and we assume that it has a simple pole. These conditions fix $\Xi(z)$. It can be expressed in terms of the theta-functions with characteristics

$$
\Xi_{k j}\left(z, u_{1}, \ldots, u_{N} ; \tau\right)=\frac{\theta\left[\begin{array}{c}
\left.\frac{k}{N}-\frac{1}{2}\right] \\
\frac{N}{2}
\end{array}\right]\left(z-N u_{j}, N \tau\right)}{\theta^{\frac{1}{N}}(z, \tau)},
$$

where

$$
\theta\left[\begin{array}{l}
a \\
b
\end{array}\right](z, \tau)=\sum_{j \in \mathbb{Z}} \exp 2 \pi i\left((j+a)^{2} \frac{\tau}{2}+(j+a)(z+b)\right) .
$$

The quasi-periodicity properties (4.3), (4.4) follow from the properties of the theta-functions

$$
\begin{aligned}
& \theta\left[\begin{array}{l}
a \\
b
\end{array}\right](z+1, \tau)=\mathbf{e}(a) \theta\left[\begin{array}{l}
a \\
b
\end{array}\right](z, \tau), \\
& \theta\left[\begin{array}{l}
a \\
b
\end{array}\right]\left(z+a^{\prime} \tau, \tau\right)=\mathbf{e}\left(-a^{\prime 2} \frac{\tau}{2}-a^{\prime}(z+b)\right) \theta\left[\begin{array}{c}
a+a^{\prime} \\
b
\end{array}\right](z, \tau) .
\end{aligned}
$$

This modification has the type $\left(\frac{N-1}{N},-\frac{1}{N}, \ldots,-\frac{1}{N}\right)$. The modification that allows to lift $\tilde{E}$ to $\operatorname{GL}(N, \mathbb{C})$ - bundle is

$$
\Xi_{1}(z)=h(z) \Xi(z)=\theta\left[\begin{array}{c}
\frac{k}{N}-\frac{1}{2} \\
\frac{N}{2}
\end{array}\right]\left(z-N u_{j}, N \tau\right),
$$

where the gauge transformation $h$ is the diagonal matrix

$$
h(z)=\theta^{\frac{1}{N}}(z, \tau) \operatorname{Id}_{N} .
$$

This modification intertwine the boundary conditions (4.2) with

$$
\rho_{a}=\mathcal{Q}, \quad \rho_{b}=\tilde{\Lambda}, \quad \tilde{\Lambda}=e^{-2 \pi i\left(\frac{z}{N}+\frac{\tau}{2 N}\right)} \Lambda .
$$

The last transformation belongs to $\operatorname{GL}(N, \mathbb{C})$. Moreover, it can be proved that

$$
\operatorname{det}\left[\frac{\Xi_{1}\left(z, u_{1}, \ldots, u_{N} ; \tau\right)}{i \eta(\tau)}\right]=\frac{\vartheta(z)}{i \eta(\tau)} \prod_{1 \leq k<l \leq N} \frac{\vartheta\left(u_{l}-u_{k}\right)}{i \eta(\tau)}
$$


where $\eta(\tau)=q^{\frac{1}{24}} \prod_{n>0}\left(1-q^{n}\right)$ is the Dedekind function $(q=\exp 2 \pi i \tau)$ and $\vartheta(z)$ is the thetafunction (2.10). Since $\vartheta(z)$ has a simple pole in $\Sigma_{\tau}$ the bundle $\tilde{E}$ is a $\operatorname{GL}(N, \mathbb{C})$-bundle of degree one. This modification provides the Symplectic Hecke correspondence between the elliptic Calogero-Moser system and the Elliptic Top.

Now consider the modification of the trivial bundle $E$ with the transition matrices (4.2) to the $\tilde{E}=E_{l}(2.11),(2.12)$, where $\mathbf{u}_{p}=\left(\tilde{u}_{1}, \tilde{u}_{2}, \ldots, \tilde{u}_{p}\right)$ is the moduli of the modified bundle. The modification takes the form

$$
\Xi_{k j}(z, \tau)=\frac{\left.\theta\left[\begin{array}{c}
\frac{k}{l}-\frac{1}{2} \\
\frac{l}{2}
\end{array}\right]\left(z-l \tilde{u}_{i}\right), l \tau\right)}{\theta^{\frac{1}{l}}(z, \tau)}, \quad(j=m p+i, m=0, \ldots, l-1) .
$$

As it was explained in Section 2 the modified bundle can be lifted to $G_{l}=\operatorname{SL}(N, \mathbb{C}) / \mathbb{Z}_{l}$-bundle, but not to $\operatorname{SL}(N, \mathbb{C})$-bundle.

\section{A $\operatorname{SL}(N, \mathbb{C})$ and $\operatorname{PSL}(N, \mathbb{C})[20,21]$}

The group $\operatorname{SL}(N, \mathbb{C})$ is an universal covering of $\operatorname{PSL}(N, \mathbb{C})$ with the center $\mathbb{Z}_{N}=\mathbb{Z} / N \mathbb{Z}$

$$
\mathrm{Id} \rightarrow \mathbb{Z}_{N} \rightarrow \mathrm{SL}(N, \mathbb{C}) \rightarrow \operatorname{PSL}(N, \mathbb{C}) \rightarrow \mathrm{Id} .
$$

Therefore $\pi_{1}(\operatorname{PSL}(N, \mathbb{C}))=\mathbb{Z}_{N}$. The both groups have the same Lie algebra $\mathfrak{G}$.

Roots and weights. The Cartan subalgebra $\mathfrak{H} \subset \mathfrak{G}$ is a hyperplane in $\mathbb{C}^{N}$

$$
\mathfrak{H}=\left\{\mathbf{x}=\left(x_{1}, \ldots, x_{N}\right) \in \mathbb{C}^{N} \mid \sum_{j=1}^{N} x_{j}=0\right\} .
$$

The simple roots $\Pi=\left\{\alpha_{k}\right\}$

$$
\alpha_{1}=e_{1}-e_{2}, \quad \ldots, \quad \alpha_{N-1}=e_{N-1}-e_{N}
$$

form a basis in the dual space $\mathfrak{H}^{*}$. Here $\left\{e_{j}\right\} j=1, \ldots, N$ is a canonical basis in $\mathbb{C}^{N}$. They generate the set of roots of type $A_{N-1}$

$$
R=\left\{\left(e_{j}-e_{k}\right), j \neq k\right\} .
$$

The root lattice $Q \subset \mathfrak{H}^{*}$ takes the form

$$
Q=\left\{\sum m_{j} e_{j} \mid m_{j} \in \mathbb{Z}, \quad \sum m_{j}=0\right\} .
$$

We identify $\mathfrak{H}^{*}$ and $\mathfrak{H}$ by means of the standard metric on $\mathbb{C}^{N}$. Then the coroot system

$$
R^{\vee}=\left\{\alpha^{\vee}(R)=\frac{2\left(\alpha^{\vee}, \beta\right)}{(\beta, \beta)} \in \mathbb{Z} \text { for any } \beta \in R\right\}
$$

coincides with $R$, and the coroot lattice $Q^{\vee}$ coincides with $Q$.

The fundamental weights $\varpi_{k},(k=1, \ldots, N-1)$ are dual to the basis of simple coroots $\Pi^{\vee} \sim \Pi\left(\varpi_{k}\left(\alpha_{k}^{\vee}\right)=\delta_{k j}\right)$

$$
\varpi_{j}=e_{1}+\cdots+e_{j}-\frac{j}{N} \sum_{l=1}^{N} e_{l},
$$




$$
\begin{aligned}
& \varpi_{1}=\left(\frac{N-1}{N},-\frac{1}{N}, \ldots,-\frac{1}{N}\right), \varpi_{2}=\left(\frac{N-2}{N}, \frac{N-2}{N}, \ldots,-\frac{2}{N}\right), \ldots, \\
& \varpi_{N-1}=\left(\frac{1}{N}, \frac{1}{N}, \ldots, \frac{1-N}{N}\right) .
\end{aligned}
$$

In the basis of simple roots the fundamental weights are

$$
\begin{aligned}
\varpi_{k}= & \frac{1}{N}\left[(N-k) \alpha_{1}+2(N-k) \alpha_{2}+\cdots+(k-1)(N-k) \alpha_{k-1}\right. \\
& \left.+k(N-k) \alpha_{k}+k(N-k-1) \alpha_{k+1}+\cdots+k \alpha_{N-1}\right] .
\end{aligned}
$$

The fundamental weights generate the weights lattice

$$
\begin{aligned}
& P \subset \mathfrak{H}^{*}, \quad P=\left\{\sum_{l} n_{l} \varpi_{l} \mid n_{l} \in \mathbb{Z}\right\}, \\
& P=\sum_{j=1}^{N} m_{j} e_{j}, \quad m_{j} \in \frac{1}{N} \mathbb{Z}, \quad m_{j}-m_{k} \in \mathbb{Z} .
\end{aligned}
$$

The weight lattice is generated by $Q$ and the vector

$$
\varpi_{1}=e_{1}-\frac{1}{N} \sum_{j=1}^{N} e_{j} .
$$

The weight lattice $P$ defines representations of $\operatorname{SL}(N, \mathbb{C})$, while $Q$ define representations of $\operatorname{PSL}(N, \mathbb{C})$.

The factor-group $P^{\vee} / Q^{\vee}\left(P^{\vee} \sim P\right)$ is the center $\mathbb{Z}_{N}$ of $\operatorname{SL}(N, \mathbb{C})$. On the other hand it can be identified with the cyclic group symmetry $e_{j} \rightarrow e_{j+1} \bmod (N)$ of the extended Dynkin graph $\Pi \cup\left(\alpha_{0}=e_{N}-e_{1}\right)$.

Characters and cocharacters. Let $\overline{\mathcal{T}}\left(\mathcal{T}_{a d}\right)$ be a Cartan torus in $\operatorname{SL}(N, \mathbb{C})(\operatorname{PSL}(N, \mathbb{C}))$. Define the groups of characters ${ }^{4}$

$$
\bar{\Gamma}=\{\bar{\chi}(x)\}=\left\{\overline{\mathcal{T}} \rightarrow \mathbb{C}^{*}\right\}, \quad \Gamma_{a d}=\left\{\chi_{a d}(x)\right\}=\left\{\mathcal{T}_{a d} \rightarrow \mathbb{C}^{*}\right\}
$$

They can be identified with lattice groups in $\mathfrak{H}^{*}$ as follows. Let $\varpi_{k}$ be a basic weight and $\phi=\left(\phi_{1}, \phi_{2}, \ldots, \phi_{N}\right), \phi_{k}=\frac{1}{2 \pi i} \ln x_{k}$. The functions

$$
\exp 2 \pi i\left(\varpi_{k} \phi\right), \quad k=1, \ldots, N-1
$$

generate a basis in $\bar{\Gamma}$. Similarly, for $\alpha_{k} \in \Pi$

$$
\exp 2 \pi i\left(\alpha_{k}, \phi\right), \quad k=1, \ldots, N-1
$$

is a basis in $\Gamma_{a d}$. Thereby, we have

$$
\bar{\Gamma}=P, \quad \Gamma_{a d}=Q .
$$

Define the dual groups of cocharacters $t(\bar{G})=\bar{\Gamma}^{*}$ and $t\left(G_{a d}\right)=\Gamma_{a d}^{*}$ as the maps

$$
t(\bar{G})=\left\{\bar{\gamma}=\mathbb{C}^{*} \rightarrow \overline{\mathcal{T}}\right\}, \quad t\left(G_{a d}\right)=\left\{\gamma_{a d}=\mathbb{C}^{*} \rightarrow \mathcal{T}_{a d}\right\} .
$$

\footnotetext{
${ }^{4}$ The holomorphic maps of the tori to $\mathbb{C}^{*}$ such that $\chi(x y)=\chi(x) \chi(y)$ for $x, y \in \mathcal{T}$.
} 
In another way

$$
t(\bar{G})=\left\{\phi \in \mathfrak{H} \mid \bar{\chi}\left(e^{2 \pi i \phi}\right)=1\right\}, \quad t\left(G_{a d}\right)=\left\{\phi \in \mathfrak{H} \mid \bar{\chi}_{a d}\left(e^{2 \pi i \phi}\right)=1\right\} .
$$

These groups are the groups of the coweight and coroot lattices

$$
t(\bar{G})=Q^{\vee} \sim Q, \quad t\left(G_{a d}\right)=P^{\vee} \sim P .
$$

The center of $\bar{\Gamma}=\operatorname{SL}(N, \mathbb{C})$ belongs to $\overline{\mathcal{T}}$ and is identified with the factor-group

$$
\begin{aligned}
\mathcal{Z}(\operatorname{SL}(N, \mathbb{C}))= & P^{\vee} / t(\bar{G}) \sim P^{\vee} / Q^{\vee} \sim \\
& \sim \pi_{1}(\operatorname{PSL}(N, \mathbb{C})) \sim t\left(G_{a d}\right) / Q^{\vee} \sim P / Q=\mathbb{Z}_{N} .
\end{aligned}
$$

Let $N=p l,(l \neq 1, N)$ and $\mathbb{Z}_{l} \subset \mathbb{Z}_{N}$ be a subgroup of $Z_{N}$. Define the factor-group

$$
G_{l}=\operatorname{SL}(N, \mathbb{C}) / \mathbb{Z}_{l}
$$

Then the center $\mathcal{Z}\left(G_{l}\right)$ of $G_{l}$ is $\mathbb{Z}_{p}$ and $\pi_{1}\left(G_{l}\right)=\mathbb{Z}_{l}$. Consider the groups of characters and cocharacters of $G_{l}$

$$
\begin{aligned}
& \Gamma\left(G_{l}\right)=\left\{\chi_{G_{l}}: \mathcal{T}\left(G_{l}\right) \rightarrow \mathbb{C}^{*}\right\}, \\
& t\left(G_{l}\right)=\left\{\gamma_{G_{l}}: \mathbb{C}^{*} \rightarrow \mathcal{T}\left(G_{l}\right)\right\},
\end{aligned}
$$

$\left(\Gamma^{*}\left(G_{l}\right)=t\left(G_{l}\right)\right)$. They are lattices in $\mathfrak{H}^{*}$ and $\mathfrak{H} Q \subset \Gamma\left(G_{l}\right) \subset P, Q^{\vee} \subset t\left(G_{l}\right) \subset P^{\vee}$. The lattice $\Gamma\left(G_{l}\right)$ is generated by the root lattice $Q$ and the vector $l \varpi_{1}$, while the lattice $t\left(G_{l}\right)$ is generated by the root lattice $Q$ and the vector $p \varpi_{1}$

$$
\Gamma\left(G_{l}\right)=l \varpi_{1} \cup Q, \quad t\left(G_{l}\right)=p \varpi_{1} \cup Q .
$$

The group $\Gamma\left(G_{l}\right)$ is the weight lattice of $G_{l}$ because highest weights of irreducible finite-dimensional representations of $G_{l}$ belong to $\Gamma\left(G_{l}\right)$.

In terms of lattices the center $\mathcal{Z}\left(G_{l}\right)$ and $\pi_{1}\left(G_{l}\right)$ take the form

$$
\begin{aligned}
& \mathcal{Z}\left(G_{l}\right) \sim P^{\vee} / t\left(G_{l}\right) \sim \Gamma\left(G_{l}\right) / Q \sim \mathbb{Z}_{p}, \\
& \pi_{1}\left(G_{l}\right) \sim t\left(G_{l}\right) / Q^{\vee} \sim P / \Gamma\left(G_{l}\right) \sim \mathbb{Z}_{l} .
\end{aligned}
$$

A subgroup ${ }^{L} G_{l} \subset \operatorname{SL}(N, \mathbb{C})$ is the Langlands dual to $G_{l}$ if

$$
t\left({ }^{L} G_{l}\right) \sim \Gamma\left(G_{l}\right) \quad\left(\Gamma\left({ }^{L} G_{l}\right) \sim t\left(G_{l}\right)\right) .
$$

It implies that

$$
\begin{aligned}
& \mathcal{Z}\left({ }^{L} G_{l}\right) \sim \mathbb{Z}_{l}, \\
& \pi_{1}\left({ }^{L} G_{l}\right) \sim \mathbb{Z}_{p} .
\end{aligned}
$$

Therefore the dual group is

$$
{ }^{L} G_{l}=G_{p} .
$$

In particular, ${ }^{L} \mathrm{SL}(N, \mathbb{C})=\operatorname{PGL}(N, \mathbb{C})$.

Affine Weil group. The affine Weyl group $W_{a}$ is a semidirect product $Q^{\vee} \rtimes W$ of the Weyl group $W$ and the group $Q^{\vee}$. It acts on $\mathfrak{H}$ as

$$
x \rightarrow x-\frac{2(\alpha, x)}{(\alpha, \alpha)} \alpha^{\vee}+k \alpha^{\vee}, \quad k \in \mathbb{Z} .
$$


Consider a semidirect product

$$
\bar{W}_{a}=P^{\vee} \rtimes W .
$$

In particular, the shift operator

$$
x \rightarrow x+\vec{m}, \quad \vec{m} \in P^{\vee}
$$

is an element from $\bar{W}_{a}$. It follows from this construction that the factor group

$$
\bar{W}_{a} / W_{a} \sim P^{\vee} / Q^{\vee} \sim \mathcal{Z}(\operatorname{SL}(N, \mathbb{C})) .
$$

Let again $N=p l$ and define a subgroup $W_{a}\left(G_{l}\right)$ of $\bar{W}_{a}$, generated by shifts from $t\left(G_{l}\right)$

$$
W_{a}\left(G_{l}\right)=t\left(G_{l}\right) \rtimes W .
$$

The factor group $W_{a}\left(G_{l}\right) / W_{a}$ is isomorphic to $t(G) / Q^{\vee}$ and in this way to $\mathcal{Z}\left({ }^{L} G_{l}\right)$.

\section{Acknowledgements}

The work was supported by grants RFBR-09-02-00393, RFBR-09-01-92437- $K E_{a}$ and NSh3036.2008.2. The work of A.Z. was also supported by the "Dynasty" fund, the President fund MK-5242.2008.2, NWO-RFBR-05-01-80006 and RFBR-09-01-93106-NCNIL . The authors are grateful for hospitality to the Max Planck Institute of Mathematics, Bonn, where the most part of this work was done.

\section{References}

[1] Levin A., Olshanetsky M., Zotov A., Hitchin systems - symplectic hecke correspondence and two-dimensional version, Comm. Math. Phys. 236 (2003), 93-133, nlin.SI/0110045.

[2] Hitchin N., Stable bundles and integrable systems, Duke Math. J. 54 (1987), 91-114.

[3] Levin A., Olshanetsky M., Zotov A., Painlevé VI, rigid tops and reflection equation, Comm. Math. Phys. 268 (2006), 67-103, math.QA/0508058.

[4] Krichever I., Vector bundles and Lax equations on algebraic curves, Comm. Math. Phys. 229 (2002), 229269, hep-th/0108110.

[5] Baxter R., Eight-vertex model in lattice statistics and one-dimensional anisotropic Heisenberg chain. I, Ann. Physics 76 (1973), 48-71.

[6] Date E., Jimbo M., Miwa T., Okado M., Fusion of the eight vertex SOS model, Lett. Math. Phys. 12 (1986), 209-215.

[7] Felder G., Conformal field theory and integrable systems associated to elliptic curves, in Proceedings of the International Congress of Mathematicians, Vols. 1, 2 (Zurich, 1994), Birkhauser, Basel, 1995, 1247-1255, hep-th/9609153.

[8] Belavin A., Dynamical symmetry of integrable system, Nuclear Phys. B 180 (1981), 189-200.

[9] Kapustin A., Witten E., Electric-magnetic duality and the geometric Langlands program, Commun. Number Theory Phys. 1 (2007), 1-236, hep-th/0604151.

[10] Levin A., Zotov A., Integrable systems of interacting elliptic tops, Teoret. Mat. Fiz. 146 (2006), 55-64 (English transl.: Theoret. and Math. Phys. 146 (2006), 45-52).

[11] Flaschka H., Newell A.C., Monodromy- and spectrum-preserving deformations. I, Comm. Math. Phys. 76 (1980), 65-116.

[12] Krichever I., The $\tau$-function of the universal Whitham hierarchy, matrix models and topological field theories, Comm. Pure Appl. Math. 47 (1994), 437-475, hep-th/9205110.

[13] Levin A., Olshanetsky M., Hierarchies of isomonodromic deformations and Hitchin systems, in Moscow Seminar in Mathematical Physics, Amer. Math. Soc. Transl. Ser. 2, Vol. 191, Amer. Math. Soc., Providence, RI, 1999, 223-262. 
[14] Arinkin D., On $\lambda$-connections on a curve where $\lambda$ is a formal parameter, Math. Res. Lett. 12 (2005), 551-565.

[15] Tong D., Quantum vortex strings: a review, arXiv:0809.5060.

Shifman M., Yung A., Supersymmetric solitons and how they help us understand non-Abelian gauge theories, hep-th/0703267.

Gorsky A., Shifman M., Yung A., $\mathcal{N}=1$ supersymmetric quantum chromodynamics: how confined non-Abelian monopoles emerge from quark condensation, Phys. Rev. D 75 (2007), 065032, 16 pages, hep-th/0701040.

[16] Popov A., Bounces/dyons in the plane wave matrix model and SU( $N)$ Yang-Mills theory, arXiv:0804.3845.

[17] Weyl A., Elliptic functions according to Eisenstein and Kronecker, Ergebnisse der Mathematik und ihrer Grenzgebiete, Band 88, Springer-Verlag, Berlin - New York, 1976.

[18] Zakharov V.E., Manakov S.V., Novikov S.P., Pitaevsky L.P., Theory of solitons. The method of the inverse scattering problem, Nauka, Moscow, 1980 (in Russian).

[19] Ward R., Integrable systems and twistors, in Integrable Systems (Oxford, 1997), Oxf. Grad. Texts Math., Vol. 4, Oxford Univ. Press, New York, 1999, 121-134.

[20] Vinberg E.B., Onishchik A.L., Seminar on Lie groups and algebraic groups, Moscow, 1988 (English transl.: Springer Series in Soviet Mathematics, Springer-Verlag, Berlin, 1990).

[21] Bourbaki N., Lie groups and Lie algebras, Chapters 4-6, Elements of Mathematics (Berlin), Springer-Verlag, Berlin, 2002. 